\title{
Reconstruction of an SSR-based Magnaporthe oryzae physical map to locate avirulence gene AvrPi12
}

\author{
Tonghui $\mathrm{Li}^{1 \dagger}$, Jianqiang Wen ${ }^{1 \dagger}$, Yaling Zhang ${ }^{1,2+}$, James Correll ${ }^{3}$, Ling Wang ${ }^{1}$ and Qinghua Pan ${ }^{1 *}(\mathbb{D}$
}

\begin{abstract}
Background: Pathogen avirulence (Avr) genes can evolve rapidly when challenged by the widespread deployment of host genes for resistance. They can be effectively isolated by positional cloning provided a robust and well-populated genetic map is available.

Results: An updated, SSR-based physical map of the rice blast pathogen Magnaporthe oryzae (Mo) has been constructed based on 116 of the 120 SSRs used to assemble the last map, along with 18 newly developed ones. A comparison between the two versions of the map has revealed an altered marker content and order within most of the Mo chromosomes. The avirulence gene AvrPi12 was mapped in a population of 219 progeny derived from a cross between the two Mo isolates CHL42 and CHL357. A bulked segregant analysis indicated that the gene was located on chromosome 6, a conclusion borne out by an analysis of the pattern of segregation shown by individual isolates. Six additional PCR-based markers were developed to improve the map resolution in the key region. AvrPi12 was finally located within the sub-telomeric region of chromosome 6, distal to the SSR locus LSM6-5.
\end{abstract}

Conclusions: The improved SSR-based linkage map should be useful as a platform for gene mapping and isolation in Mo. It was used to establish the location of AvrPi12, thereby providing a starting point for its positional cloning.

Keywords: Magnaporthe oryzae, SSR physical map, Avirulence gene, AvrPi12, Genetic and physical mapping

\section{Background}

The pathogen responsible for the highly damaging disease of rice known as blast is the filamentous ascomycete Magnaporthe oryzae (Mo) [1, 2]. Deployment of host genes conferring resistance is widely recognized as the most environmentally benign and cost-effective means of its control [3-5]. However, the effectiveness of most blast resistance genes ceases after a few seasons, as a result of the emergence of pathogen races in which the matching avirulence (Avr) gene has mutated to virulence [6-10]. A better understanding of the mode of evolution of pathogen Avr genes should aid in forming an effective strategy for resistance gene deployment.

\footnotetext{
* Correspondence: panqh@scau.edu.cn

†Tonghui Li, Jianqiang Wen and Yaling Zhang contributed equally to this work.

'State Key laboratory for Conservation and Utilization of Subtropic

Agrobioresurces, Guangdong Provincial Key Laboratory for Crop Molecular Breeding, College of Agriculture, South China Agricultural University, Guangzhou 510642, China

Full list of author information is available at the end of the article
}

Positional cloning has proven to be an effective means of isolating both host resistance and pathogen Avr genes [10-14]. The method relies on the prior establishment of a comprehensive linkage map [11, 15]. The effort to develop such a linkage map for $\mathrm{Mo}$, begun in the 1990s, and by 2007 had delivered one based on SSR (simple sequence repeats; also called microsatellite) markers, taking advantage of the acquisition of the genome sequence of Mo isolate 70-15 (MG5; http://www.ncbi.nlm.nih.gov/bioproject/13840; $[15,16])$. In the meantime, improvements have been made to this genome sequence (MG8; www.ncbi.nlm.nih.gov/assembly/GCF_000002495.2/); [17, 18]), which now allows for an update of the $M o$ linkage map.

Pi12, a gene which conditions blast resistance, was initially identified in the African cultivar Moroberekan [19, 20], but is also effective in parts of East Asia [21-23]. Here, the updated Mo physical map was used to identify the genomic location of the Avr gene matching Pi12, the most important blast resistance gene harbored by 
Moroberekan, as a prelude to undertaking its positional cloning.

\section{Methods \\ The Mo mapping population}

Isolates CHL357 and CHL42 were collected from diseased plants in, respectively, Jiangsu and Yunnan provinces (China). The two isolates were crossed with one another in vitro using a published protocol [24, 25], and the resulting single-spored progeny were incubated for the first 7 days at $25{ }^{\circ} \mathrm{C}$ and thereafter at $20{ }^{\circ} \mathrm{C}$ under continuous illumination provided by dark-blue fluorescent light. Single ascospores were randomly selected [24], generating a population of 219 viable isolates, which were stored on dry filter papers [25]. This set of progeny represented the mapping population used to determine the genetic location of AvrPi12.

\section{Reconstruction of SSR physical map for Mo genome}

The SSR loci previously developed based on the genome of the version MG5 [15] were reassembled onto the version MG8 by aligning their primer sequences using the Blast algorithm (http://www.ncbi.nlm.nih.gov/BLAST), even though some primer sequences were slightly improved for better detection. A number of additional SSR assays were subsequently developed to fill gaps resulting from monomorphism between the mapping population parents. The old markers (prefixing with MS) integrated into the new version of the SSR physical map were re-prefixed with SM, and the new ones further post fixed with A and B, if any. The Avr genes isolated were also included in the SSR physical map with their sequence information. The methods used to identify SSRs, to design primers and to deploy the PCR assays followed those described by Feng et al. [15].

\section{Gene analysis}

A monogenic line with resistance gene Pi12, IRBL12-M, the universal susceptible cultivar Lijiangxintuanheigu (LTH; also the recipient for a set of monogenic lines;), and other 10 monogenic cultivars/ lines with the respective resistance genes, Pia, Pii, Pik, Pik-p, Pit, Pi2, Pi11, Pib, Pita, Pita-2 [26] (data will be shown elsewhere), were used as the host cultivars in this study. Seeds of 12 cultivars were separately sown in a plastic pot (diameter $17 \mathrm{~cm}$, height $9.5 \mathrm{~cm}$ ). Three to five plants per cultivar were used for inoculation. The methods used for Mo inoculation and disease scoring followed those described elsewhere [27]. Each isolate was tested at least twice, and the highest disease score recorded was adopted as the true score. The pattern of segregation for Avr (disease rating of $0-2$ ) to Pi12 across the mapping population was expected to be $1: 1$ since $M o$ is haploid [10, 24,
25], and the observed ratio was tested against this expectation using a standard $x^{2}$ test.

\section{Linkage mapping}

Initially, the bulked segregant analysis (BSA) [28] approach was taken to identify the genetic location of AvrPi12. The two necessary contrasting bulk DNAs were formed by creating an equimolar mixture of DNA extracted from either ten isolates which were all avirulent or ten which were all virulent when inoculated on IRBL12-M. The two bulk DNAs were tested with 132 genomically well dispersed SSR assays. SSRs identified as being potentially linked to AvrPi12 according to the BSA assay were then used to genotype individual members of the mapping population. To refine the map location, this step was repeated using a number of newly developed PCR-based markers (single nucleotide polymorphisms and indels) known to lie within the critical genomic region. Since $M o$ is a haploid organism, the recombination frequency between adjacent markers is given by the ratio between the number of recombinants and the total number of individuals tested $[10,15,24]$. As the relevant recombination frequencies were all below $4.5 \%$, they were directly equivalent to cM [24]. Since the markers used for mapping had been placed by inspection of the genome sequence of $M o$ isolate $70-15$, it was possible to convert the genetic map into a physical one. The disordered genomic region between the donor isolate and the reference isolate 70-15, if any, was adjusted by the actual recombinants detected at the respective loci. The methods used to extract DNA from the Mo isolates, to develop the new markers and to perform genotyping followed those given by Ma et al. [24].

\section{Results}

The updated SSR-based Mo physical map

Of the 120 SSR markers used to develop the original linkage map, 116 were retained in the updated version (Fig. 1; Additional file 1: Table S1; also see [15]). The four old markers, MS4-6, 4-7, 6-15, and 7-2, were ruled out, as their positions were missing in the MG8 (MS4-6, 7-2), or their assays proved to be non-robust (MS4-7, 6-15). A further 18 SSR loci were added to the set of 116. A comparison between the two versions of the physical map showed that just two of chromosomes (3 and 7) were almost maintained with respect to their SSR content, although their marker order was altered in both cases (see SM and MS marker codes in Additional file 1: Table S1). The marker content of the updated version of chromosome 1 was almost the same as that of the previous version of chromosome 2 , and similarly for the new chromosome 5/previous chromosome 6 and the new chromosome 6/previous chromosome 4 . The 


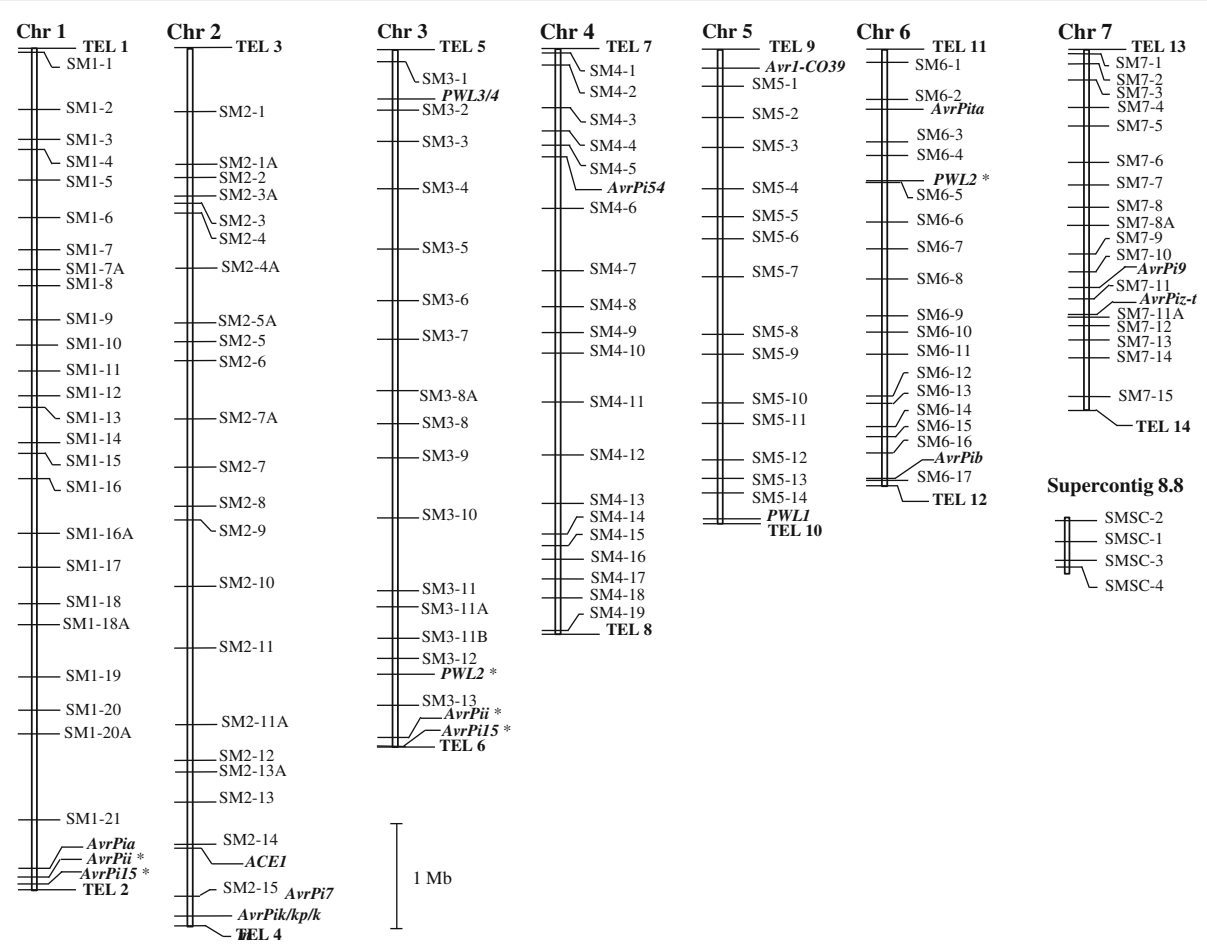

Fig. 1 Updating the Mo SSR-based linkage map, based on the current version of the reference genome sequence of isolate 70-15 (MG8; https:// www.ncbi.nlm.nih.gov/assembly/GCF_000002495.2/). The map was constructed using 116 of the 120 markers reported in [15], along with 18 newly developed ones (see also Additional file 1: Table S1). The telomeres were indicated by boldface, and the cloned Avr genes were integrated into the map with their sequences. PWL1, and PWL3/4 [35], PWL2 [36], AvrPita [31], ACE1 [37], AvrPiz-t [43], AvrPia [33, 38], AvrPii and AvrPik/kp/km [33], AvrPi54 [44], AvrPi15 [24], AvrPi7 [25]. AvrPi15, AvrPii, Avr1-CO39 and PWL2 located on double positions were marked by *. AvrPia, AvrPii and Avr1-CO39, which were absent on 70-15, were landed by their flanking sequences

updated version of the chromosome 2 map incorporated SSRs previously allocated to chromosomes 1 and 4; while the new version of chromosome 4 combined markers previously associated with chromosomes 1, 3 and 5. An additional chromosome, referred to here as "Supercontig 8.8", harbored markers previously assigned to chromosome 7 . Thus the updated version of the linkage map comprised eight chromosomes, according to the MG8 genome (Fig. 1).

\section{Avirulence inheritance}

The parental isolates CHL42 and CHL357 were, respectively, avirulent and virulent on IRBL12-M, while they were both virulent on LTH (Additional file 2: Figure S1). When the 219 progeny isolates were tested individually for their avirulence/virulence on IRBL12-M, avirulence and virulence segregated in a $1: 1$ ratio $\left(111: 108, x^{2}=\right.$ $0.02, P<0.10)$. This result further suggested that the progeny population tested consisted of random ascospore isolates. The progeny population was, therefore, recognized as an appropriate mapping population for AvrPi12, which is responsible to the resistance gene Pi12 carried by IRBL12-M.

\section{Chromosome landing}

To search for the chromosomal location for the AvrPi12 locus, a total of 132 SSR markers covering the whole genome of $\mathrm{Mo}$, were screened by BSA assay, of which 90 showed a polymorphism between two parental isolates (Fig. 2 and Additional file 3: Figure. S2). The results showed that markers on chromosomes $1-5$ and 7 as well as Supercontig 8.8 were unlikely as candidate ones linked to the locus (Additional file 3: Figure. S2). In contrast, a series of markers located on chromosome 6 showed concurrent polymorphisms for both parental and bulk pairs (Fig. 2). Three polymorphic markers, SM6-12, SM6-16, and SM6-17, were selected for tentative validation with the progeny isolates ranged from $\# 103$ to \#135. The results showed that all the three markers were linked to the AvrPi12 locus (Fig. 3). Taking into a consideration that the AvrPi12 locus might be rearranged by some segments from other chromosomes [10], four putative polymorphic markers, SM2-1, SM215, SM4-2, and SM7-14, were tested with the progeny isolates ranged from \#1 to \#035, individually. The results showed that all the four markers were not linked to the AvrPi12 locus (Additional file 4: Figure. S3). Taken 


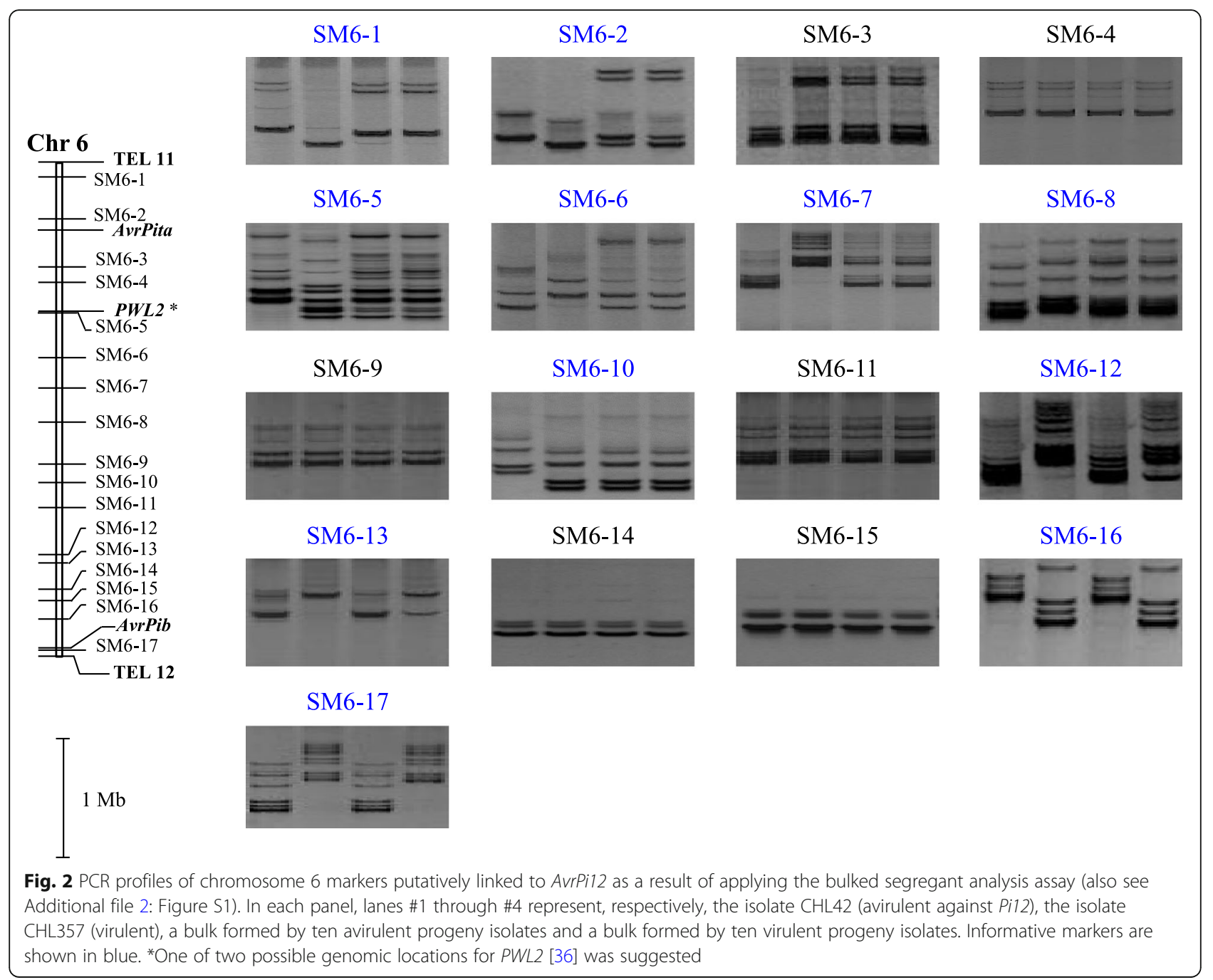

together, the data supported a location for AvrPi12 on chromosome 6.

\section{Gene location}

Three above-mentioned markers were further tested with the rest progeny isolates in the mapping population. The results showed that 47, 22, and 6 recombinants, respectively, detected at markers SM6-12, SM616, and SM6-17 in the first round of linkage analysis (Fig. 4). Because all the recombinants from SM6-12, through SM6-16, and to SM6-17 overlapped, and the last one is close to the telomere of Mo, TEL12, the AvrPi12 locus was defined between SM6-17 and TEL12 (Fig. 4). To construct a fine map of the locus, six additional markers were de novo developed for the second round of linkage analysis (Table 1). The results showed that there were $20,16,13$, and 9 recombinants, detected at markers, LSM6-4, LSM6-6, LSM6-9, and LSM6-1, respectively, indicating that these markers were located to an interval between markers SM6-16 and SM6-17. As to marker LSM6-2, there were 23 recombinants, suggesting a genetic inversion between markers SM6-16 (22 recombinants) and LSM6-2 was occurring in the parental genome (Fig. 4). The six recombinants detected between AvrPi12 and LSM6-5 were the same as those detected between AvrPi12 and SM6-17, thereby narrowing the genetic window harboring AvrPi12 to the interval between LSM6-5 and TEL12. Each genetic distance was determined by recombinants occurred in the region between adjacent markers, which was shown above the map in cM. The estimated physical distances between the various linked markers were mostly derived from the isolate 70-15 genome sequence; the exceptions involved the inferred inversion between SM6-16 and LSM6-2, and the interval between LSM6-5 and TEL12, which is of uncertain length, given that telomeric regions of many plant pathogens appear to be 

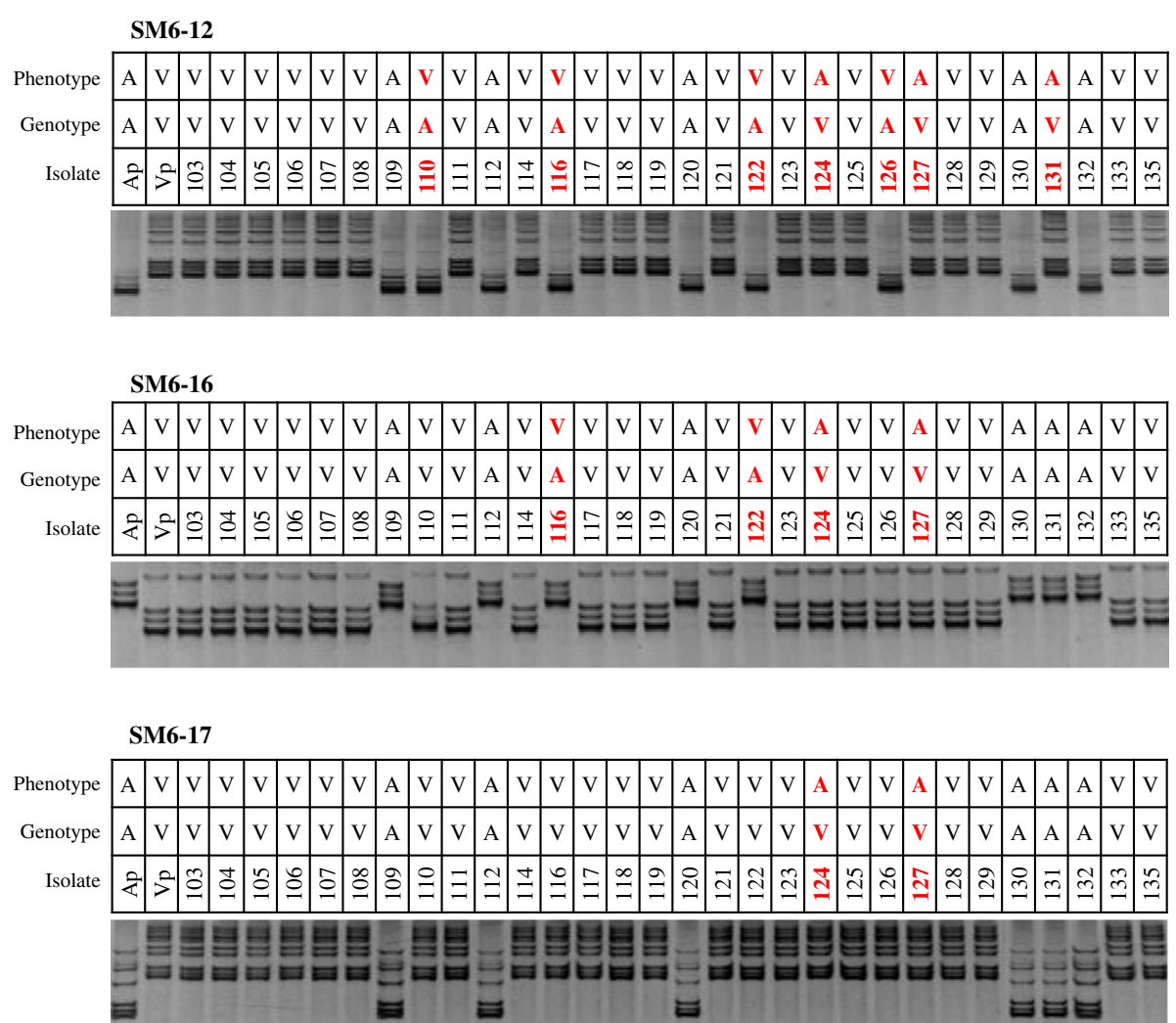

Fig. 3 PCR profiles of 30 progeny isolates and their parental isolates derived from three linked markers. Phenotypes: A, avirulent; $V$, virulent. Genotypes: $A$, the same with $A$ parent (Ap); $V$, the same with $V$ parent $(V p)$. The recombinants were in red

hypervariable $[24,29,30]$. As per the reference genomic sequence of $70-15$, in this region, there were 12 candidate genes predicted for AvrPi12 (Additional file 5: Table S2). Intriguingly, there was not any one meeting criterions for both secretion and effector, indicating that AvrPi12 might be located on a specific interval, which was absent in the genome of 70-15.

\section{Discussion}

The updated Mo physical map can serve as a platform for gene mapping and cloning

The first whole genome sequence of $M o$ was released by IRGBC in 2003 (MG5; [16]). Since then, a critical effort has been made by the IRGBC in collaboration with the Broad Institute, USA, to improve the accuracy and integrity of the reference sequence that led to the release a

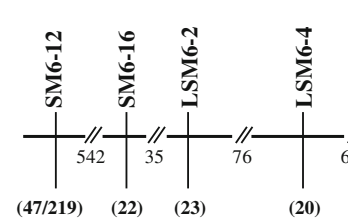

b

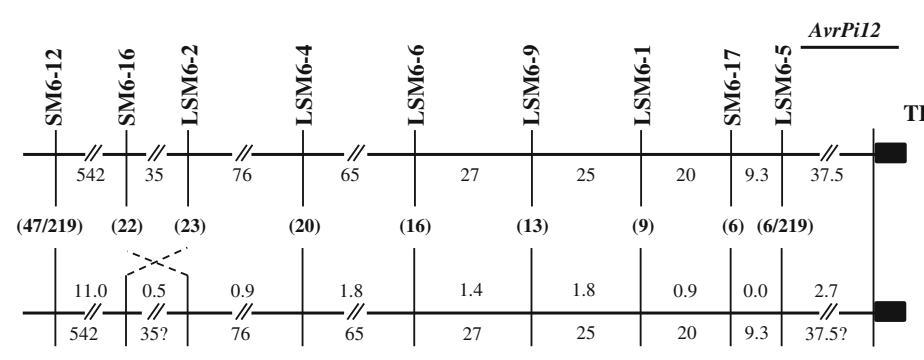

(

(Physic distance, kb)

Fig. 4 Genetic and physical maps of the AvrPi12 locus. a A physical map of markers used for chromosome walking to AvrPi12 locus based on the reference genome of isolate 70-15. b Genetic and physical map of the AvrPi12 locus. The numbers shown below the map indicate distance between adjacent markers. Recombinants detected at each marker was shown in parenthesis, and the respective genetic distance between adjacent markers was shown above the map in CM (not shown to scale). The physical distances in the parental genomes of both isolates CHL42 and CHL357 were generally referred to those of 70-15, where the physical distances of two intervals were questioned, one was in an inversion between markers SM6-16 and LSM6-2, and another in the telomeric region between markers LSM6-5 and TEL12 
Table 1 PCR-based markers linked to the AvrPi12 locus mapping to Mo chromosome 6

\begin{tabular}{|c|c|c|c|c|c|}
\hline Marker $^{a}$ & Type $^{\text {b }}$ & Primer sequence $\left(5^{\prime} \rightarrow 3^{\prime}\right)^{c}$ & ${\text { Genomic position }(b p)^{d}}^{d}$ & $\operatorname{Tm}\left({ }^{\circ} \mathrm{C}\right)^{\mathrm{e}}$ & Size $(b p)^{f}$ \\
\hline \multirow[t]{2}{*}{ SM6-12 } & SSR & F: CGTATTCTTGGCTGAGTGGC & 6: 3283269 & 60 & 293 \\
\hline & & R: GCCGACGACCTGTGTGATAC & & & \\
\hline \multirow[t]{2}{*}{ LSM6-2 } & InDel & F: GCGAGAGTTTGACTGATGTTTG & 6: 3857026 & 60 & 86 \\
\hline & & R: ATCCACCCAAGCTITCGTTTAG & & & \\
\hline \multirow[t]{2}{*}{ SM6-16 } & SSR & F:TGATACTAACTCCTCCTCCCAAAAC & 6: 3826452 & 57 & 159 \\
\hline & & R: CAGTCACGGTCTCCTAAGCC & & & \\
\hline \multirow[t]{2}{*}{ LSM6-4 } & InDel & F: GCAGTAGGTGAATTGTCTCGGT & 6: 3932760 & 58 & 124 \\
\hline & & R: AGTCACCCCTACTCTGTTGTTGT & & & \\
\hline \multirow[t]{2}{*}{ LSM6-6 } & SNP & F: ACCGAGTTTAGTGTTITGGATGA & 6: 4011510 & 58 & 165 \\
\hline & & R: TCGAAGGTTTATGGTGCCAAT & & & \\
\hline \multirow[t]{2}{*}{ LSM6-9 } & SNP & F: CCACCCTGTGTCGTACTTCAATT & 6: 4040268 & 58 & 112 \\
\hline & & R: AAGATTGGTGGCCTGTCGTT & & & \\
\hline \multirow[t]{2}{*}{ LSM6-1 } & InDel & F: ATACCAGAACAAATTGCAAACAGC & 6: 4065773 & 60 & 46 \\
\hline & & R: GTTACACCGAGGAACTTGCTTG & & & \\
\hline \multirow[t]{2}{*}{ SM6-17 } & SSR & F: GGCGGCAAGTCGCTGAAGG & $6: 4086125$ & 58 & 330 \\
\hline & & R: GAGTTTGAGACCTTGCGATT & & & \\
\hline \multirow[t]{2}{*}{ LSM6-5 } & SSR & F: GAGACGATGGGCCTCTAGCA & 6: 4096513 & 59 & 143 \\
\hline & & R: CTCCACGACGGTATGTTTGC & & & \\
\hline
\end{tabular}

${ }^{\mathrm{a}} \mathrm{SM}$ markers were the basic markers as shown in Fig. 1 and Additional file 1: Table S1, and LSM markers were de novo developed markers specific for the AvrPi12 locus

${ }^{\mathrm{b}} \mathrm{SSR}$, simple sequence repeat; SNP, single nucleotide polymorphism; InDel, insertion-deletion

${ }^{\mathrm{C}} \mathrm{F}$, forward; $\mathrm{R}$, reverse

${ }^{d}$ Genomic positions were based on the latest version of the reference genome sequence of isolate $70-15$

(MG8; https://www.ncbi.nlm.nih.gov/assembly/GCF_000002495.2/)

${ }^{\mathrm{e}}$ All runs began with one cycle at $94^{\circ} \mathrm{C}$ for $3 \mathrm{~min}$, followed by 30 cycles at $94^{\circ} \mathrm{C}$ for $30 \mathrm{~s}, 55-62^{\circ} \mathrm{C}$ (annealing temperature, $\mathrm{Tm}$ ) for $30 \mathrm{~s}$, and $72^{\circ} \mathrm{C}$ for $1-1.5$ min; with a final extension at $72^{\circ} \mathrm{C}$ for $7 \mathrm{~min}$

${ }^{\mathrm{f}}$ Amplicons obtained in the mapping population were separated by electrophoresis on $8 \%$ or $10 \%$ polyacrylamide gels

of the latest version of the reference genome sequence (MG8; $[17,18])$. Comparison of the two versions of the SSR physical maps established based on MG5 and MG8 revealed that there were certain changes in both the content and the order of each chromosomal marker set (Fig. 1; Additional file 1: Table S1; also see [15]). Such dramatic changes between the two versions of the SSR physical maps that, in turn, indicated that the new one would serve as a more robust platform for gene mapping and cloning. It is, however, necessary to take into account a careful consideration for the specificities of the pathogen genomes, such as variability and plasticity, when adopted any reference sequence and linkage/physical map for individual studies [10, 14, 25, 31-34]. As to the linkage/physical maps of the AvrPi12 locus, there were two genomic intervals being evaluted in the current study, one for genetic inversion between markers SM6-16 and LSM6-2, and another for the telomeric region between LSM6-5 and TEL12. A close understanding of the dynamics of genomic structure surrounding the target locus is crucial for chromosome walking to the target region $[10,14,25,32,34]$.
The physical maps of AvrPi12 can serve as a start point for deciphering molecular mechanism underlying durable resistance of Moroberekan

In the updated SSR physical map, some 14 Avr gene loci including three $P W L$ loci, where some target genes have been isolated, have been mapped based on their sequence information (Fig. 1). Ten loci were located in the sub-telomeric and telomeric regions: PWL1, and PWL3/ 4 [35], PWL2 [36], AvrPita [31], ACE1 [37], AvrPia [33, 38], AvrPii [33], AvrPik/kp/km [33], Avr1-CO39 [39], and $A v r P i b$ [10]. A similar situation was also identified in other pathosystems $[29,30,40]$. It was well recognized that the allocation of Avr genes in the dynamic telomeric regions was indeed one of the most forceful strategies for the pathogens to confront resistance gene-driven positive selection $[7,10,30,31,41]$. As a result, it is in turn a bigger challenge for chromosome walking to the target locus in the hypervariable region $[10,24,25,41,42]$. However, the substantial genomic sequence resources developed for species belonging to the Magnaporthaceae family can facilitate an in silico reconstruction of the telomeric region of a given isolate 
(www.ncbi.nlm.nih.gov/assembly/organism/318829/latest/; [10, 24, 25]).

The blast resistance of Moroberekan appears to have help up very well despite its cultivation over many years across a large area of West Africa [19]. Its durability is thought to be due its harboring of the three major genes Pi12, Pi5 and Pi7, in combination with an unknown number of minor genes $[19,20]$. Consideration of its resistance spectra has concluded that the key determinant of its high level of blast resistance across Africa has been Pi12, while its interaction with the other two major genes may be as important in East Asia [21-23]. Because AvrPi5 and AvrPi7 are known to represent alleles of, respectively, AvrPii and AvrPik (unpublished data), the isolation of the AvrPi12 should allow for studies on the gene-for-gene network underlying the durable resistance of Moroberakan. Based on the genetic and physical maps of the AvrPi12 locus reported here, the next step forward will be to initiate a chromosome walk from LSM6-5 to TEL12.

\section{Conclusions}

The current version of the Mo genome sequence has been exploited to update its SSR-based physical map. A comparison between the updated and the original versions of the physical maps has revealed alterations with respect to both marker content and marker order within most of the chromosomes. AvrPi12 was mapped to chromosome 6 using a population of 219 progeny isolates derived from the cross CHL42 x CHL357. More detailed mapping showed that the gene lays in a telomeric region distal to the SSR locus LSM6-5; the latter marker could serve as the starting point for a chromosome walk to the target locus.

\section{Additional files}

Additional file 1: Table S1 Primer pair sequences, SSR motifs, genomic positions and PCR conditions of the 134 SSR markers used to construct the linkage map (DOCX 51 kb)

Additional file 2: Figure S1 The distinct reactions derived from the parental isolates each interacted with the monogenic line carrying Pi12, IRBL12-M, and its susceptible recipient, LTH. (PDF 3420 kb)

Additional file 3: Figure S2 PCR profiles of the first 30 progeny isolates and their parental isolates which derived from four putative polymorphic markers those selected from BSA analysis. (PPTX 199 kb)

Additional file 4: Figure S3 Bulked segregant analysis PCR profiles for the 117 SSR markers mapping to the non-critical chromosomes 1-5, 7 and Supercontig 8.8 (17 markers on chromosome 6 were shown in Fig. 2). (PPTX $1322 \mathrm{~kb}$ )

Additional file 5: Table S2 Candidate genes for AvrPi12 that were predicted in the target region flanked by ZSM6 and TEL12 (DOCX $22 \mathrm{~kb}$ )

\section{Abbreviations}

Avr: Avirulence; BSA: Bulked segregant assay; InDel: Insertion-deletion; IRBGP: International Rice Blast Genome Project; LTH: Lijiangxintuanheigu;
Mo: Magnaporthe oryzae; SNP: Single nucleotide polymorphism; SSR: Simple sequence repeats

\section{Acknowledgments}

We thank Profs. Y. Zhu at Yunnan Agricultural University, and X. Zhen at Nanjing Agricultural University for providing the parental isolates, CHL42 and CHL357, respectively.

\section{Funding}

This work was supported by the National Key R\&D Project (2016YDF0100601), the National Transgenic Research Project (2016ZX08001002), the National Natural Science Foundation of China (U1131003), and the Natural Science Foundation of Heilongjiang Province (QC2011C046). Each funding body has not role in the design of the study and collection, analysis, and interpretation of data and in writing the manuscript.

\section{Availability of data and materials}

All data generated or analysed during this study are included in this published article and its supplementary information files. Collection of both plant and pathogen materials were complied with institutional, national, and international guidelings.

\section{Author's contributions}

Project conception (QP), mapping population construction (YZ, LW), phenotyping (JW, TL, LW, QP), genotyping (TL, JW, YZ), data analysis ( $T L, Y Z$, JW, QP), map reconstruction ( $T L, J W, Q P)$, manuscript preparation $(Q P, J C)$. All authors read and approved the final manuscript.

Ethics approval and consent to participate Not applicable.

\section{Competing interests}

The authors declare that they have no competing interests.

\section{Publisher's Note}

Springer Nature remains neutral with regard to jurisdictional claims in published maps and institutional affiliations.

\section{Author details}

${ }^{1}$ State Key laboratory for Conservation and Utilization of Subtropic Agrobioresurces, Guangdong Provincial Key Laboratory for Crop Molecular Breeding, College of Agriculture, South China Agricultural University, Guangzhou 510642, China. ${ }^{2}$ College of Agronomy, Heilongjiang Bayi Agricultural University, Daqing 163319, China. ${ }^{3}$ Department of Plant Pathology, University of Arkansas, Fayetteville, AR 72701, USA

Received: 21 February 2018 Accepted: 21 May 2018

Published online: 31 May 2018

References

1. Kiyosawa S. Genetics and epidemiological modeling of breakdown of plant disease resistance. Annu Rev Phytopathol. 1982;20:93-117.

2. Ou S. Pathogen variability and host resistance in rice blast disease. Annu Rev Phytopathol. 1980;18:167-87.

3. He X, Liu X, Wang L, Wang L, Lin F, Liao Y, et al. Identification of the new recessive gene pi55(t) conferring resistance to Magnaporthe oryzae. Sci China Life Sci. 2012:55:141-9.

4. Garrett K, Andersen K, Asche F, Bowden R, Forbes G, Kulakow P, et al. Resistance genes in global crop breeding networks. Phytopathology. 2017; 107:1268-78.

5. Zhang $Y$, Zhu Q, Yao Y, Zhao Z, Correll J, Wang L, et al. The race structure of the rice blast pathogen across southern and northeastern China. Rice. 2017;10:46

6. Flor H. Current status of the gene-for-gene concept. Annu Rev Phytopathol. 1971;9:275-96.

7. Wu W, Wang L, Zhang S, Li Z, Zhang Y, Lin F, et al. Stepwise arms race between AvrPik and Pik alleles in the rice blast pathosystem. Mol PlantMicrobe Interact. 2014;27:759-69.

8. Zhai C, Zhang Y, Yao N, Lin F, Liu Z, Dong Z, et al. Function and interaction of the coupled genes responsible for the Pik-h encoded blast resistance of rice. PLoS One. 2014;9:e98067. 
9. Wu J, Kou Y, Bao J, Li Y, Tang M, Zhu X, et al. Comparative genomics identifies the Magnaporthe oryzae avirulence effector AvrPi9 that triggers Pi9-mediated blast resistance in rice. New Phytol. 2015;206:1463-75.

10. Zhang S, Wang L, Wu W, He L, Yang X, Pan Q. Function and evolution of Magnaporthe oryzae avirulence gene AvrPib responding to the rice blast resistance gene Pib. Sci Rep. 2015;5:11642.

11. Kaye C, Milazzo J, Rozenfeld S, Lebrun M. Tharreau D (2003) the development of simple sequence repeat markers for Mgnaporthe grisea and their integration into an established genetic linkage map. Fungal Genet Biol. 2003:40:207-14.

12. Peter J, Cnudde F, Gerats T. Forward genetics and map-based cloning approaches. Trend in Plant Sci. 2003;8:484-91.

13. Liu W, Liu J, Ning Y, Ding B, Wang X, Wang Z, et al. Recent progress in understanding PAMP- and effector-triggered immunity against the rice blast fungus Magnaporthe oryzae. Mol Plant. 2013;6:605-20.

14. Praz C, Bourras S, Zneg F, Sanchez-Martin J, Menardo F, Xue M, et al. AvrPm2 encodes an RNase-like avirulence effector which is conserved in the two different specialized forms of wheat and rye powdery mildew fungus. New Phytol. 2016;213:1301-14.

15. Feng S, Ma J, Lin F, Wang L, Pan Q. Construction of an electronic physical map of Magnaporthe oryzae using genomic position-ready SSR markers. Chinese Sci Bull. 2007;52:3346-54.

16. Dean R, Talbot N, Ebbole D, Farman M, Mitchell T, Orbach M, et al. The genome sequence of the rice blast fungus Magnaporthe grisea. Nature. 2005;434:980-6.

17. Mao $X$, Jiang $H$, Wang $Y$, Zhang Z, Chai R, Wang J, et al. Comparison on genome sequence of Magnaporthe oryzae in different assembly databases. Chn J Rice Sci. 2013;27:425-33.

18. Okagaki L, Nunes C, Sailsbery J, Clay B, Brown D, John T, et al. Genome sequences of three phytopathogenic species of the Magnaporthaceae family of fungi. G3. 2015;5:2539-45.

19. Wang G, Mackill D, Bonman J, McCouch S, Champoux M, Nelson RRFLP. Mapping of genes conferring complete and partial resistance to blast in a durably resistant rice cultivar. Genetics. 1994;136:1421-34.

20. Inukai $T$, Zeigler $R$, Sarkarung $S$, Bronson M, Dung L, Kinoshita $T$, et al. Development of pre-isogenic lines for rice blast-resistance by markeraided selection from a recombinant inbred population. Theor Appl Genet. 1996;93:560-7.

21. Zhu Q. Comparison of pathotype structures of Magnaporthe oryzae populations collected in south and northeast of China. Guangzhou: Master Thesis of South China Agricultural University; 2014. (In Chinese with English Abstract)

22. Kawasaki-Tanaka A, Hayashi N, Yanagihara S, Fukuta Y. Diversity and distribution of rice blast (Pyricularia oryzae Cavara) races in Japan. Plant Dis. 2016;100:816-23.

23. Mutiga S, Rotich F, Ganeshan V, Mwongera D, Mgonja E, Were V, et al. Assessment of the virulence spectrum and its association with genetic diversity in Magnaporthe oryzae populations from sub-Saharan Africa. Phytopathology. 2017;107:852-63.

24. Ma J, Wang L, Feng S, Lin F, Pan Q. Identification and fine mapping of AvrPi15, a novel avirulence gene of Magnaporthe grisea. Theor Appl Genet. 2006;113:875-83.

25. Feng S, Wang L, Ma J, Lin F, Pan Q. Genetic and physical mapping of AvrPi7, a novel avirulence gene of Magnaporthe oryzae using physical position-ready markers. Chinese Sci Bull. 2007;52:903-11.

26. Tsunematsu H, Yanoria M, Ebron L, Hayashi N, Ando I, Kato H, et al. Development of monogenic lines of rice for blast resistance. Breeding Sci. 2000;50:229-34.

27. Pan $\mathrm{Q}$, Wang $\mathrm{L}$, Ikehashi $\mathrm{H}$, Tanisaka T. Identification of a new blast resistance gene in the indica rice cultivar Kasalath using Japanese differential cultivars and isozyme markers. Phytopathology. 1996;86:1071-5.

28. Michelmore R, Paran I, Kesseli R. Identification of markers linked to disease-resistance genes by bulked segregant analysis: a rapid method to detect markers in specific genomic regions by using segregating populations. Proc Natl Acad Sci U S A. 1991;88:9828-32.

29. Bishop R, Gobright E, Nene V, Morzaria S, Musoke A, Sohanpal B. Polymorphic open reading frames encoding secretory proteins are located less than 3 kilobases from Theileria parva telomeres. Mol Biochem Parasital. 2000;110:359-71.

30. Barry J, Ginger M, Burton P, McCulloch R. Why are parasite contingency genes often associated with telomeres? Int J Parasital. 2003;33:29-45.
31. Orbach M, Farrall L, Sweigard J, Chumley F, Valent B. A telomeric avirulence gene determines efficacy for the rice blast resistance gene Pi-ta. Plant Cell. 2000;12:2019-32.

32. Luo C, Yin L, Koyanagi S, Farman M, Kusaba M, Yaegashi H. Genetic mapping and chromosomal assignment of Magnaporthe oryzae avirulence genes AvrPik, AvrPiz, and AvrPiz-t controlling cultivar specificity on rice. Phytopathology. 2005;95:640-7.

33. Yoshida K, Saitoh H, Fujisawa S, Kanzaki H, Matsumura H, Yoshida K, et al. Association genetics reveals three novel avirulence genes from the rice blast fungal pathogen Magnaporthe oryzae. Plant Cell. 2009;21:1573-91.

34. Bao J, Chen $M$, Zhong Z, Tang W, Lin L, Zhang X, Jiang $H$, et al. PacBio sequencing reveals transposable elements as a key contributor to genomic plasticity and virulence variation in Magnaporthe oryzae. Mol Plant. 2017;10:1465-8

35. Kang S, Sweigard J. Valent B (1995) the PWL host specificity gene family in the blast fungus Magnaporthe grisea. Mol Plant-Microbe Interact. 1995:8:939-48.

36. Sweigard J, Carroll A, Kang S, Parrall L, Chumley F, Valent B. Identification, cloning, and characterization of PWL2, a gene for host species specificity in the rice blast fungus. Plant Cell. 1995;7:1221-33.

37. Böhnert H, Fudal I, Dioh W, Tharreau D, Notteghem J, Lebrun M. A putative polyketide synthase/peptide synthetase from Magnaporthe grisea signals pathogen attack to resistant rice. Plant Cell. 2004;16:2499-513.

38. Miki S, Matsui K, Kito H, Otsuka K, Ashizawa T, Yasuda N, et al. Molecular cloning and characterization of the AVR-Pia locus from a Japanese field isolate of Magnaporthe oryzae. Mol Plant Pathol. 2009:10:361-74.

39. Ribot C, Césari S, Abidi I, Chalvon V, Bournaud C, Vallet J, et al. The Magnaporthe oryzae effector AVR1-CO39 is translocated into rice cells independently of a fungal-derived machinery. Plant J. 2013;74:1-12.

40. Scherf A, Figueiredo L. Freitas-junior L (2001) Plasmodium telomeres: a pathogen's perspective. Curr Opin Microbiol. 2001:4:409-14.

41. Farman L, Kim Y. Telomere hypervariability in Magnaporthe oryzae. Mol Plant Pathol. 2005:6:287-98.

42. Gao W, Khang C, Park S, Lee Y, Evolution KS. Organization of a highly dynamic, subtelomeric helicase gene family in the rice blast fungus Magnaporthe grisea. Genetics. 2002;162:103-12.

43. Li W, Wang B, Wu J, Lu G, Hu Y, Zhang X, et al. The Magnapporthe oryzae avirulence gene AvrPiz-t encodes a predicted secreted protein that triggers the immunity in rice mediated by the blast resistance gene Piz-t. Mol PlantMicrobe Interact. 2009;22:411-20.

44. Ray S, Singh P, Gupta D, Mahato A, Sarkar C, Rathour R, et al. Analysis of Magnaporthe oryzae genome reveals a fungal effector, which is able to induce resistance response in transgenic rice line containing resistance gene, Pi54. Front Plant Sci. 2016;7:1140.

\section{Ready to submit your research? Choose BMC and benefit from:}

- fast, convenient online submission

- thorough peer review by experienced researchers in your field

- rapid publication on acceptance

- support for research data, including large and complex data types

- gold Open Access which fosters wider collaboration and increased citations

- maximum visibility for your research: over $100 \mathrm{M}$ website views per year

At BMC, research is always in progress.

Learn more biomedcentral.com/submissions 